\title{
EL VIDEOJUEGO COMO ESPEJO DE LA SOCIEDAD CONTEMPORÁNEA
}

\section{VIDEO GAMES AS A MIRROR OF THE CONTEMPORARY SOCIETY}

\author{
Antonio César Moreno Cantano \\ Universidad Complutense de Madrid. Madrid / España \\ antmor03@ucm.es \\ https://orcid.org/0000-0003-1008-2831 \\ Alberto Venegas Ramos \\ Universidad de Murcia. Murcia / España \\ alberto.venegasr@um.es \\ https://orcid.org/0000-0002-5621-7749
}

Recibido/Received: 15/09/2020

Modificado/Modified: 06/10/2020

Aceptado/Accepted: 09/11/2020

\section{RESUMEN}

El presente texto adquiere la forma de introducción del monográfico dedicado a Videojuegos como representación de la problemática social. Nos planteamos la importancia creciente de este formato para posicionar al jugador frente a gran número de problemáticas actuales, que cuestionan y critican aspectos de la realidad política, social o económica. Tal y como han planteado diferentes investigadores, tales como Daniel Muriel, Alberto Murcia o Ian Bogost, mediante este tipo de simulaciones y experiencias, el juego puede contribuir a confrontar nuestra identidad y empatizar con aquello que se nos muestra a través de la pantalla. Se produce un efecto de espejo, en el que las mecánicas y reglas de algunos de los más conocidos títulos videolúdicos, pueden plantearnos dilemas morales que van más allá de ganar o perder una partida.

\section{PALABRAS CLAVE}

Videojuegos; problemáticas actuales; representación social; identidad; empatía.

\section{SUMARIO}

1. Introducción. 2. El juego, la pantalla y la vida real. 3. Los videojuegos como reflejo de la realidad. 4. Los videojuegos como medio de interacción social. 5. El yo y el otro en el videojuego. 6. Los videojuegos: objeto de estudio. 7. Diferencia entre el videojuego y lo real. 4. A modo de síntesis y esbozo futuro. Bibliografia.

\begin{abstract}
The present text takes the form of an introduction to the monograph dedicated to Videogames as a representation of social problems. We consider the growing importance of this format to position the player in front of a great number of current problems, which question and criticize aspects of political, social or economic reality. As different researchers, such as Daniel Muriel, Alberto Murcia or Ian Bogost, have proposed, through this type of simulations and experiences, the game can contribute to confront our
\end{abstract}


identity and empathize with what is shown to us through the screen. A mirror effect is produced, in which the mechanics and rules of some of the best known video game titles can pose moral dilemmas that go beyond winning or losing a game.

\section{KEYWORDS}

Video games; Current problems; Social representation; Identity; Empathy.

\section{CONTENTS}

1. Introduction. 2. Games, Screens and real Life. 3. Video Games as a Reflection of Reality. 4. Video Games as a Means of social Interaction. 5. The Self and the Other in the Video Game. 6. Video Games: Object of Study. 7. Difference between the Video Game and the real Thing. 4. By Way of Synthesis and future Outline. References.

\section{INTRODUCCIÓN}

"Estudiar el videojuego hoy es estudiar nuestra contemporaneidad". Esta frase fue pronunciada por la filósofa Eurídice Cabañes (2019) a raíz de la entrevista que la Fundación Telefónica llevó a cabo para promocionar la exposición "Videojuegos. Los dos lados de la pantalla". La hemos traído a colación para iniciar la presentación de este novedoso, y necesario, monográfico centrado en la representación de la problemática social a través de este complejo medio de expresión y comunicación social. Y, además, nos permite iniciar una reflexión sobre el juego de espejos al que hacía referencia el título del citado evento.

\section{EL JUEGO, LA PANTALLA Y LA VIDA REAL}

Al posicionarnos ante nuestro monitor, entramos en un entorno virtual que debe su razón de ser a un tiempo y espacio específico, cuyos componentes (diseño gráfico, narrativa, mecánicas, temática, sonido, interacción) son incomprensibles sin el bagaje de la sociedad en que se llevó a cabo. Los fenómenos sociales, políticos o económicos de ese momento y ese lugar sirven de fuente para construir, de objeto de reflexión, de límite autoimpuesto o de problemáticas que se reproducen y repiten en el medio. El videojuego recoge ese universo simbólico de la sociedad, perpetuándolo y limitándolo. Desgranar su esencia, mensajes, dinámicas, contribuyen de manera cualitativa a una mejor comprensión de nosotros mismos y cómo nos proyectamos en nuestras creaciones digitales. Su carácter transversal, mutable, en constante actualización, lo ha convertido en un "ser" permeable que copa cada vez mayor protagonismo en las agendas políticas, económicas, culturales o educativas, de diferentes actores nacionales, regionales, ONGs u otro tipo de organismos. Se ha "ganado" un privilegiado posicionamiento público, que está eliminando (o al menos dejando en segundo plano) los estereotipos y clichés violentos y aditivos que antaño eran su primera seña de identificación ante el gran público.

\section{LOS VIDEOJUEGOS COMO REFLEJO DE LA REALIDAD}

La participación del videojuego como reflejo de los problemas políticos y sociales actuales ha experimentado una gran evolución en los últimos meses, en especial con acontecimientos como el Brexit (que han puesto en valor el deseo de determinadas identidades y colectivos de 
no ser absorbidos por estructuras globales), el confinamiento debido a la COVID-19 o los recientes protestas en Hong Kong. Símbolos propios de estos productos digitales culturales, como por ejemplo el ganso de Untitled Goose Game (House House, 2019), enarbolado por parte de la izquierda británica en algunas manifestaciones, son muestras del cariz político e ideológico que el videojuego está adquiriendo. Sus desarrolladores se posicionaron de manera decidida contra Boris Johnson utilizando la imagen de este palmípedo al que añadieron una rosa roja en alusión a los laboristas. A través de las cuentas oficiales en Twitter de relevantes títulos y compañías, como Bloodborne (From Sotfware, 2015) y Annapurna Interactive (responsable de Outer Wilds, entre otros), el mundo del videojuego se ha expresado de manera contundente contra el racismo tras la muerte en Minneapolis de George Floyd. Como manifestó Renee Gittins, directora ejecutiva de la International Game Developers Association: "Los desarrolladores de juegos creamos arte que impulsa a las personas con emociones y empatía, podemos usar nuestros talentos no solo para mejorar nuestra industria, sino también para mejorar el mundo".

No sorprende, por tanto, que algunos de los títulos de mayor éxito a nivel mundial, como por ejemplo Animal Crossing: New Horizons (Nintendo, 2020), se hayan convertido en una plataforma de reivindicación social y promoción política. Si en un principio este título dio cabida a las reivindicaciones de los manifestantes de Hong Kong contra Pekín, en la pasada campaña electoral entre el Presidente Donald Trump y el candidato Joe Biden ha servido de espacio virtual para captar votos entre la población más joven. Organizaciones como NextGen America (de signo Demócrata y financiada por el multimillonario Tom Steyer) han modificado los entornos de Animal Crossing para conectar con posibles apoyos. De esa misma tendencia, la congresista por Nueva York, Alexandria Ocasio-Cortez, creó su propia cabaña para atraer seguidores y poder interactuar con ellos en un medio virtual. $\mathrm{Y}$ en fecha tan reciente como octubre, apareció un avatar que se identificaba plenamente con los rasgos y símbolos del aspirante Demócrata, como sus famosas gafas de sol.

\section{LOS VIDEOJUEGOS COMO MEDIO DE INTERACCIÓN SOCIAL}

No es desdeñable tampoco la dimensión de los videojuegos como elemento de interacción social, tómese el caso de los e-sports. El tradicional estereotipo de jugador solitario, y aislado de la sociedad se desmonta en este caso. El videojuego ya no se concibe como un mero entretenimiento individual, sino que gracias al avance de las TIC son posibles dinámicas en la que varios jugadores de distintos puntos del mundo jueguen, e interaccionan entre sí. Pero los $e$-sports van mucho más allá, jugar se convierte en un evento social, y más allá de ello en un deporte de competición (Vera, 2015). Además de las consecuencias a nivel social cabe valorar también el fuerte impacto económico, con unas cifras de visitas a las competiciones de 453 millones de usuarios, ingresando la industria de los e-sports más de 1.000 millones de dólares (Cristòfol et al., 2020). Los videojuegos han entrado en la industria del espectáculo para quedarse, por lo que también es interesante tener en cuenta su papel como actividad económica en el mundo audiovisual.

\section{EL YO Y EL OTRO EN EL VIDEOJUEGO}

Pero el videojuego no solo participa de elementos políticos y económicos. Habla de nosotros y por eso mismo nosotros debemos hablar con ellos. Pone a los jugadores en la piel de los 
demás, permitiéndoles experimentar el mundo desde sus perspectivas. Sin embargo, los jugadores no sienten exactamente lo que los otros, después de todo, son una experiencia mediada, no la experiencia en sí misma; pero permite a los jugadores al menos conectarse con las realidades de los demás de diferentes maneras, tal y como se defiende en la obra Video Games as Culture (2019), de Daniel Muriel y Garry Crawford. El ser humano actual pide algo más que saber, quiere vivir, probar, interactuar, ya sea con el mundo real o virtual, no en vano en muchas ocasiones este no es más que un mero espejo pixelado de aquello que nos rodea. Pero como producto permeable, también es poroso a los elementos más negativos de nuestro ser, reproduciendo en su interior y en su proceso creativo todos los males de nuestra realidad social contemporánea. De esta manera, a finales de octubre del presente año, los desarrolladores del videojuego Cyberpunk 2077 (CD Project), como Andrzej Zawadzki, fueron amenazados de muerte por los fan del mismo porque se habían retrasado veintiún días en su lanzamiento.

Desde el problema y preocupación más candente de nuestra complejidad a la experiencia más minoritaria (pero no por ello menos relevante) tienen cabida en formato pixelado. Sirvan de ejemplo Night in the Woods (Infinite Fall, 2017), sobre la precariedad juvenil y la despoblación de las zonas rurales; Flower (Thatgamecompany, 2009) y su mensaje ecocrítico; Emporium (Tom Kitchen, 2017) y su reflexión acerca del suicidio en nuestra sociedad; Fran Bow (Killmonday Games, 2018) y los desórdenes mentales o Keys of a Gamespace (Sébastien Genvo, Expressive Gamestudio, 2011) y nuestra relación con el pasado y la memoria para forjar nuestra identidad en el presente. Todos ellos rodean el tema sobre el que nacen o lo abordan directamente, todos ellos establecen un diálogo con nuestra realidad y nuestra problemática cotidiana.

\section{LOS VIDEOJUEGOS: OBJETO DE ESTUDIO}

Con este objetivo doble, conocer cómo los videojuegos abordan o evitan los problemas actuales de la sociedad y qué dicen de ella aquellos que hablan o callan sobre distintas problemáticas, nace este monográfico. A través de los diversos artículos presentados, pretendemos conformar un corpus heterogéneo y diverso capaz de abordar aspectos como el precariado, el suicidio, la muerte, la soledad, el envejecimiento de la población, la inmigración, la identidad, la memoria, el derecho a reclamar un futuro mejor o a imaginarlo... Temas que condicionan nuestra realidad y se convierten en retos para nuestra vida durante la modernidad. Si nos acogemos a la categorización de Zygmunt Bauman (2002), está determinada por su naturaleza "líquida". El cambio se diluye rápidamente a través de todos los sectores de la vida de una manera global, interconectada, no existen moldes de ninguna naturaleza capaz de evitar la cultura de lo instantáneo.

El videojuego, dentro de su doble naturaleza lúdico-técnica, es una de las más claras manifestaciones de esta nueva sociedad, caracterizada por la democratización del acceso a la información y a las producciones culturales. Explicaba Ortega y Gasset en su célebre obra ¿Qué es Filosofía? que gracias al conocimiento podíamos obtener cierto orden dentro del caos de la vida. El videojuego, como simulador de la realidad lleva implícito en su diseño, reglas, mecánicas, narrativas, todos los males / buenaventuras de la realidad presente, y contribuye en gran medida a "reordenar", poner en orden la complejidad del mundo que nos rodea desde un sentido lúdico. Apuntaban Jordi Vallverdú y Yosuke Nakano en Ensayos y errores (AnaitGames, 2019) que "no hay nada que sea más real que un videojuego", pues "si nuestra propia identidad se define al vivir en entornos sociales, entonces la realidad de los entornos 
sociales que se desarrollan en la red y los videojuegos se mezcla con nuestra corporalidad y mentes". De la misma opinión, el crítico y profesor Ian Bogost expresaba en Persuasive Games (2007):

Los videojuegos son un medio expresivo. Representan cómo funcionan los sistemas reales e imaginarios. Invitan a los jugadores a interactuar con esos sistemas y a formarse juicios sobre ellos. Como parte del proceso continuo de entender este medio y llevarlo más lejos como jugadores, desarrolladores y críticos, debemos esforzarnos por entender cómo construir y criticar las representaciones de nuestro mundo en forma de videojuego.

Estamos, por tanto, ante la emergencia de lo que Larissa Hjorth (2011) ha denominado como "tecno-culturas donde los nuevos dispositivos tecnológicos no son algo meramente funcional, sino que realmente operan tanto en el nivel simbólico como en los niveles materiales de nuestras vidas cotidianas". Como apuntan dos de los autores de este monográfico (Puente y Sequeiros, 2019) en un estudio previo:

Al reaprender los mecanismos del nuevo juego y reconstruir la mentalidad de los usuarios, se continúa reconfigurando la subjetividad y el cuerpo de cada jugador. Al hacerlo, el medio se convierte en un espacio de interacción y disputa que permite que los jugadores se introduzcan de lleno en un escenario dramático compartido e imbricado en la cotidianeidad, donde representan los distintos roles que les corresponden.

Todo ello nos lleva a plantearnos una cuestión clave, “¿se puede entender lo humano desde lo lúdico?" (Murcia, 2020: 16-22). De poco sirve dirigir nuestros esfuerzos a intentar explicar cómo los videojuegos representan las principales problemáticas actuales si no valoramos si estas analogías entre lo ficcional y lo real tienen un impacto significativo en los jugadores.

\section{DIFERENCIA ENTRE EL JUEGO Y LO REAL}

¿Se es mejor politólogo, historiador, sociólogo o antropólogo solo por jugar? En este camino son básicas las mecánicas del juego, aquello que podemos hacer o no dentro del mismo y plantearnos el porqué de una acción, las consecuencias de las mismas y extrapolarlas al plano de la realidad. Existe todo un elenco de juegos (como algunos de los que se analizan en este monográfico) que plantean diferentes tipos de "elecciones narrativas", con una mayor o menor significación en el jugador. La investigadora Clara Fernández Vara establece una breve taxonomía de las mismas entre las que sobresalen los llamados "supuestos dilemas morales" y los "dilemas auténticos". Esta última categorización es la más interesante, porque ninguna de las opciones que se nos ofrezca tiene una solución óptima y cada ventaja tiene su inconveniente. Se plantean dilemas que revelan la complejidad del mundo, en el que las soluciones para "ganar" el juego también significa hacer cosas moralmente reprochables (Fernández, 2020).

Un ejemplo muy ilustrativo sobre las implicaciones morales que pueden acarrear nuestros actos para conseguir la victoria dentro de un videojuego es: Raid Gaza! (Raitendo, 2008). Enmarcado en el sempiterno conflicto israelí-palestino, el videojuego toma posición y construye un marco crítico con la política judía. Desde el punto de vista estético, el juego abre 
con una imagen de Ehud Olmert, antiguo primer ministro israelí y la frase "los parámetros de una solución unilateral pasan por maximizar las bajas judías y reducir las bajas palestinas". Aparecen grandes letras cuando se toca algún objetivo que da "bonus"; estaciones de policía u hospitales. Destaca el texto que aparece al solicitar ayuda a los países occidentales; un teléfono rojo expresa el desprecio hacia los acuerdos de Oslo o los Derechos Humanos. "Oh, ¿necesitas más dinero? ¡Por qué no lo dijiste antes! Te voy a transferir unos cuantos millones para, eh... cómo llamarlo, 'ayuda humanitaria'? Ja Ja Ja!’. Raid Gaza! explicita su marco persuasivo una vez finalizados los tres minutos de la partida. A través de una caricatura de Ehud Olmert se presentaba la relación entre bajas palestinas y bajas judías (estas producidas por ocasionales misiles que nunca llegan a impactar edificios de Israel, sino que caen en el campo) que ha alcanzado el jugador, para después compararla con un estudio realizado por Naciones Unidas en el que se explicaba que por un judío muerto en el conflicto fallecían paralelamente 25 palestinos.

\section{A MODO DE SÍNTESIS Y ESBOZO FUTURO}

Este monográfico se inserta en estas interpretaciones y marcos teóricos, y pretende profundizar y debatir acerca de cómo los videojuegos se relacionan con nuestra sociedad y tratan, o callan, los problemas que nos rodean. Para ello hemos recurrido a un equipo multidisciplinar y transversal, que desde diferentes enfoques (Historia, Periodismo, Filosofía, Sociología, Comunicación Audiovisual) y metodologías, quieren aproximar este producto cultural y sus potencialidades representativas y comunicativas a un gran público, tanto especializado como genérico, con el propósito último de que el ocio videolúdico sea no solo una válvula de escape, sino también un elemento de valoración y estudio de nuestro mundo.

Moreno y Vilajosana pondrán sus miras en el análisis de la personalista obra de Tom Kitchen, un desarrollador británico, que a través del título autobiográfico Emporium (2017) mostró la complejidad de decisiones que pueden conducir al suicidio. El proceso de diseño de este juego, a su vez, tuvo un fuerte componente terapéutico para afrontar sus dificultades emocionales, a la par que un ejemplo para otros usuarios con el que compartir y superar la misma dolorosa experiencia: depresión, soledad y muerte.

Sobre esta última cuestión versa su texto Gisbert, que recurre a la semiótica para el estudio de la identidad y muerte en el afamado videojuego Final Fantasy IX (Squaresoft, 2000) y cómo las formas que en él adoptan tienen un reflejo en la sociedad actual. Sobre el concepto de la muerte siguen reflexionando Garrido y Martín a partir de A Mortician's Tale (DaRienzo, 2017), título que se inspira en el proyecto The Order of the Good Death, que promueve hablar abiertamente sobre el fin de la vida a través de cadáveres, entierros y otros aspectos que suelen ser silenciados en la actualidad al ser considerados un tabú.

Por su parte, Sequeiros y Puente profundizan en la capacidad que presentan los videojuegos, que como dispositivos sociotécnicos no solo reproducen estructuras, hegemonías y repertorios compartidos, sino que son capaces de permear y afectar a nuestras identidades. Mientras jugamos aprendemos y nos embebemos de un espacio social que nos reconfigura como sujetos políticos, tal y como se comprueba mediante las narrativas y mecánicas de títulos como Los Sims (Maxis, 2000), Papers, Please (Lucas Pope, 2013) o Detroit: Become Human (Quantic Dream, 2018), entre otros. Aparecen en muchos de estos casos las "elecciones morales", que nos llevan al dilema de avanzar en el juego sin tener en cuenta las consecuencias vitales que acarrean para los personajes allí insertos: impedir el paso fronterizo, aunque eso suponga la posterior muerte de un refugiado, por ejemplo. Es en este género de mecánicas donde nuestra 
identidad, nuestros valores, son puestos en solfa a través del diseño ludotécnico.

En unos ejes temáticos similares se posiciona Venegas y su exhumación del concepto de memoria en el videojuego de historia. Plantea la disyuntiva entre el acto de construir o reconstruir el pasado, reflejar el dolor de las víctimas o ignorarlo y convertir los hechos pretéritos en un "parque de atracciones" supeditado a la estetización y al consumismo de la imagen. Mediante un potente marco teórico (de Walter Benjamin a Tzvetan Todorov), este axioma será ejemplificado en el estudio de videojuegos como Trough the Darkest of Times (Paintbucket Games, 2020), Wolfenstein II: The New Collosus (MachineGames, 2017) o Venti Messi (We Are Muesli, 2016). Imaginar un mundo mejor ha sido siempre una aspiración del ser humano, pero la triste realidad presente, con todas las cadenas que la sujetan, nos llevan a configurar en nuestras mentes escenarios venideros de signo distópico, en los que la libertad ha sucumbido a los intereses de regímenes tecnológicos de signo autoritario. Este género distópico, sustentado en una gruesa literatura (Dick, Bradbury, Howey), ha avanzado imparablemente en el mundo del videojuego.

Suárez disecciona los elementos de The Last of Us II (Naughty Dog, 2020) y profundiza en la relación entre Joel y Ellie para proyectar en este espacio apocalíptico los problemas que no hemos sabido solucionar en el presente. Y para ello la ciudadanía, la multitud, podemos protestar, movilizarnos y reivindicar nuestros derechos ante el Poder.

El filósofo Antonio Flores aborda como esta colectividad se convierte en determinados títulos, como Tonight we riot (Pixel Pusher Union 512, 2020) o Riot: Civil Unrest (Leonard Menchiari/IV Productions, 2019), en un personaje jugable y medita sobre la significación política que adquirimos como individuo inseparable de una colectividad, ya sea en la Plaza Tahrir (Egipto y la Primavera Árabe) o la Plaza Mayor (Protestas del 15-M en Madrid). No sorprende que ante este sombrío panorama un sector de la población pueda sufrir cierto tipo de enfermedades, absorbidos por el ritmo imparable e inmisericordioso del ultra-neo-liberalismo occidental.

Paredes aporta en su texto lucidez y visibilidad a ese enemigo invisible como son las enfermedades mentales, que como parte de nuestro ser también merecen la atención de la industria del videojuego. Hay un esfuerzo creciente para que el usuario pueda empatizar y sensibilizarse a estigmas como la esquizofrenia -The Town of Light (LKA, 2017)-, la psicosis -Hellblade: Senua's Sacrifice (Ninja Theory, 2018)- o la ansiedad / depresión -Celeste (Matt Makes Games, 2018). Lo real no es incompatible con el ocio, es más, hay un nutrido grupo de jugadores / as que buscan la inmersión virtual como mecanismo de reflexión sobre los males que gravitan sobre nuestro mundo.

Este monográfico otorgará un lugar privilegiado a cómo el videojuego intenta dar respuesta a muchos de estos pesares, balanceándose en esa delgada línea que hay entre lo ficticio y lo tangible.

\section{BIBLIOGRAFÍA}

Bauman, Z. (2002) Modernidad Líquida. México: Fondo de Cultura Económica.

Bogost, I. (2007) Persuasive Games: the expressive power of video games, Cambridge, Massachusetts: The MIT Press.

Cabañes, E. (2019) Videojuegos y contemporaneidad. Espacio Fundación Telefónica, en https://espacio.fundaciontelefonica.com/noticia/videojuegos-y-contemporaneidad-un-todo-paraeuridice-cabanes/

Cristòfol, F.; Martínez-Ruiz, Á.; Román-Navas, I. y Cristófol-Rodríguez, C. (2020) Evolución de las 
estrategias de patrocinio en los esports en España: 2013-2021. Ámbitos. Revista Internacional de Comunicación, (48), 188-204, doi: 10.12795/Ambitos.2020.i48.XX

Fernández, C. (2020) "Elecciones. Diseño narrativo de decisiones y ramificaciones", en V. Navarro

(Coord.), Pensar el juego. 25 caminos para los Game Studies, Asociación Shangrila Textos Aparte, pp. 64-71.

Hjorth, L. (2011) Games and Gaming (an Introduction to New Media). New York: Berg.

Murcia, A. (2020) “Analogía. ¿Se puede entender lo humano desde lo lúdico?, en V. Navarro (Coord.), Pensar el juego. 25 caminos para los Game Studies, Asociación Shangrila Textos Aparte, pp. 16-22.

Ortega y Gasset, J. ([1929] 2015) ¿Qué es Filosofía? Madrid: Alianza Editorial.

Puente Bienvenido, H. y Sequeiros Bruna, C. (2019) Goffman y los videojuegos: Una aproximación sociológica desde la perspectiva dramatúrgica a los dispositivos videolúdicos. Revista Española De Sociología, 28 (2), en https://recyt.fecyt.es/index.php/res/article/view/68948

Vallverdú, J. y Nakano, Y. (2019) "No hay nada que sea más real que un videojuego", en D. Ferragut \& A. García (Eds.), Ensayo y errores. Arte, Ciencia y Filosofía en los videojuegos, Anait Games, pp. $135-156$.

Vera, J. (2015) La dimensión social de los videojuegos' online': de las comunidades de jugadores a los 'esports'. Index. comunicación: Revista científica en el ámbito de la Comunicación Aplicada, 5(1), 3951, en https://journals.sfu.ca/indexcomunicacion/index.php/indexcomunicacion/article/view/173/163

\section{Breve currículo:}

\section{Antonio César Moreno Cantano}

Profesor Asociado del Departamento de Relaciones Internacionales e Historia Global de la Universidad Complutense de Madrid. Acreditación ANECA para la figura de Profesor Ayudante y Contratado Doctor. Miembro del Proyecto de Investigación Historia y Videojuegos II. Conocimiento, aprendizaje y proyección del pasado en la sociedad digital (HAR2016-78147-P), de la Universidad de Murcia y de GEINTEA de la Universidad Complutense de Madrid, entre otros. En la actualidad está investigando sobre el empleo de serious / newsgames para la representación de los principales conflictos internacionales y su utilización con fines didácticos.

\footnotetext{
Alberto Venegas Ramos

Licenciado en Historia por la Universidad de Extremadura (2006-2011), Máster en Educación Secundaria Obligatoria por la Universidad de Extremadura (2011-2012), Máster universitario en métodos y técnicas de investigación histórica por la UNED (2012-2013), profesor de Educación Secundaria Obligatoria (desde 2014) y doctorando por la Universidad de Murcia con la tesis doctoral: Historia y Videojuegos: La Segunda Guerra Mundial en la cultura y la sociedad digital contemporánea (2017-2019). Miembro del grupo de investigación del Ministerio de Ciencia, Innovación y Universidades Historia y Videojuegos 2.0: conocimiento, aprendizaje y proyección del pasado en la sociedad digital (HAR2016-78147-P) y autor del libro Pasado interactivo: memoria e historia en el videojuego (Sans Soleil, 2020).
} 